\title{
A Major QTL Controlling Earliness of Fruit Maturity Linked to the Red leaf/Red flesh Trait in Apple cv. 'Maypole'
}

\author{
Takuya Morimoto $^{1}$, Yuko Hiramatsu ${ }^{1}$ and Kiyoshi Banno ${ }^{2 *}$ \\ ${ }^{1}$ Graduate School of Agriculture, Shinshu University, Minamiminowa, Nagano 399-4598, Japan \\ ${ }^{2}$ Faculty of Agriculture, Shinshu University, Minamiminowa, Nagano 399-4598, Japan
}

\begin{abstract}
Anthocyanins, which contribute red coloration to apple skin, are recognized for their antioxidant properties. Some apple cultivars also accumulate anthocyanins in fruit flesh. In this study, we analyzed the inheritance of coloration traits in fruit skin, fruit flesh, and leaves as well as their candidate gene, and searched for quantitative trait loci (QTLs) for fruit maturity linked to the red flesh trait in 'Maypole', using a 'Fuji' $\times$ 'Maypole' $F_{1}$ population. Phenotypic segregation in the $F_{1}$ population indicated that red skin, striped skin, red flesh, and red leaf traits are each controlled by separate single dominant genes and that red leaves co-segregated with red flesh. Of these, a striped skin trait derived from 'Fuji' corresponded to the $M d M Y B A$ genotype, known to regulate anthocyanin synthesis in fruit skin, suggesting that this genotype may be responsible for fruit skin coloring patterns. The red leaf/red flesh trait derived from 'Maypole' co-segregated with the MdMYB10 R6 promoter. $M d M Y B A$ and $M d M Y B 10$ were located in the same region at the bottom of linkage group (LG) 9 on the same genetic material, supporting that these genes are allelic. Analysis of the relationship between red coloration and fruit maturity revealed that fruits of red-fleshed progeny tended to mature earlier than those of white-fleshed progeny. A major QTL accounting for $35.6 \%$ of the total variance (logarithm of odds [LOD] $=8.94$ ) was detected near the MdMYB10 locus in 'Maypole', indicating that the factor controlling earliness of fruit maturity is tightly linked to the red leaf/red flesh trait.
\end{abstract}

Key Words: fruit maturity, Malus $\times$ domestica, MdMYB10, QTL analysis, red flesh trait.

\section{Introduction}

Apple (Malus $\times$ domestica Borkh.) is one of the most economically important fruit crops in many temperate regions. World-wide commercial apple production was more than 70 million tons in 2010 (FAOSTAT, http:// faostat.fao.org/, December 26, 2012). Red coloration of apple skin, which is due to anthocyanins, is an important determinant of fruit marketability and an indicator of nutritional value (Eberhardt et al., 2000). In some cultivars, anthocyanins may also accumulate in the fruit flesh, although cultivars that possess this "red flesh trait", including 'Red Field', 'Almata', and 'Geneva', have not to date been marketed commercially due to poor fruit quality (e.g., high astringency, acidity, and small size). Red pigmentation in fruit flesh tissue is a highly valuable trait; however, its novel color may increase consumer appeal and red-fleshed apples can be used in processed

Received; October 5, 2012. Accepted; December 28, 2012.

* Corresponding author (E-mail: bannoki@shinshu-u.ac.jp). goods such as juice and pies. Moreover, since each coloration trait in fruit skin and flesh is reported to be controlled by a single dominant gene (Chagne et al., 2007; Takos et al., 2006), these traits can be readily exploited in breeding programs using marker-assisted selection.

Extensive molecular research has been carried out on red coloration in many crops. Expression of structural genes involved in the anthocyanin biosynthetic pathway is known to be differentially regulated by MYB transcription factors. In apples, three different R2R3type MYB transcription factors controlling anthocyanin synthesis coded by MdMYB10, MdMYB1, and MdMYBA have been identified (Ban et al., 2007; Espley et al., 2007; Takos et al., 2006). The MdMYB10 protein regulates red pigmentation in fruit flesh and leaves, and its expression level is correlated with anthocyanin content in flesh tissue (Espley et al., 2007). Espley et al. (2009) have further shown that red leaf/red flesh varieties contain tandem repeats of a 23-bp sequence in the MdMYB10 promoter region. This repeat motif is a target 
of the MdMYB10 protein itself, and the number of repeat units correlates with an increase in MdMYB10 protein trans-activation. MdMYB1 and MdMYBA, which share identical nucleotide sequences, have been shown to regulate anthocyanin synthesis in fruit skin, and are induced by low temperature and UV-B (Ban et al., 2007; Takos et al., 2006; Ubi et al., 2006). Although MdMYB10 and $M d M Y B 1$ are expressed in different organs, their coding sequences are $98 \%$ identical (Ban et al., 2007). In addition, $M d M Y B 10$ and $M d M Y B A$ have been mapped to the same region of LG 9 in different cultivars (Ban et al., 2007; Chagne et al., 2007). Lin-Wang et al. (2010) also showed that MdMYB10 and MdMYBA are likely alleles of each other using gene-specific primers. Furthermore, the chromosomal region of these $M d M Y B$ genes in the apple genome (Velasco et al., 2010) seems to contain only one candidate gene (Lin-Wang et al., 2011).

There have been many reports on the regulatory network of anthocyanin synthesis by $M d M Y B$ genes and mapping of the candidate genes for coloration traits. Very little is known, however, about the effects of MdMYB10 expression or the red flesh trait on apple growth and fruit quality traits, such as tree vigor, fruit maturity, and fruit size. Recently, Gao et al. (2011) reported that MdMYB10 over-expression in Arabidopsis enhanced osmotic tolerance through increased concentration of defense factors such as flavonoids, chlorophyll, and proline, but its effect on apples is unknown. Various quantitative trait loci (QTLs) have been identified during the search for the genetic mechanisms underlying apple growth and fruit quality traits, but only white-fleshed cultivars were used in those studies (Kenis and Keulemans, 2007; Kenis et al., 2008; King et al., 2001; Liebhard et al., 2003). To accelerate the development of efficient breeding programs for apple cultivars with the red flesh trait, elucidation of the various characteristics associated with the red flesh trait or MdMYB10 expression in the fruit flesh is therefore needed. For example, many red-fleshed varieties that have been released to date, including 'Geneva', 'Redfield', and 'Almata', are known to be early maturing cultivars, suggesting a relationship between the red flesh trait and earliness.

In 1999 , we generated $F_{1}$ populations from crosses between 'Fuji' or 'Tsugaru' and 'Maypole', the latter of which possesses the red flesh trait. Most of the resulting progeny have now fruited, allowing us to investigate fruit traits of interest. The objectives of the present study were to: (1) analyze the relationship between MdMYB10 and $M d M Y B A$ along with their association with coloration phenotypes, and (2) identify the QTL controlling earliness of fruit maturity linked to the red flesh trait in 'Maypole'.

\section{Materials and Methods}

\section{Plant materials}

Two cross combinations between apple cultivars 'Fuji' or 'Tsugaru' and 'Maypole' were carried out in 1999, resulting in 79 and 31 progeny in the 'Fuji' $\times$ 'Maypole' and 'Tsugaru' $\times$ 'Maypole' $F_{1}$ populations, respectively. 'Maypole', obtained from 'Wijcik McIntosh' $\times$ 'Baskatong', has red leaves and red flesh inherited from 'M. pumila var. niedzwetzkyana' (ancestor of 'Baskatong') and a columnar habit derived from 'Wijcik McIntosh'. Progeny used in this study were grown on their own roots and planted $0.5 \mathrm{~m}$ apart in rows separated by $2.0 \mathrm{~m}$ at the experimental farm of the Laboratory of Pomology, Faculty of Agriculture, Shinshu University, Nagano, Japan. Orchard management was performed according to commercial practices utilized in the southern part of Nagano Prefecture.

For DNA extraction, fresh young leaves of parental cultivars and 'Fuji' $\times$ 'Maypole' progeny were collected and stored at $-85^{\circ} \mathrm{C}$. Genomic DNA was extracted from $0.5 \mathrm{~g}$ of leaves (fresh weight) using a Plant Genomic DNA Maxi Extraction Kit (Viogene, New Taipei, Taiwan). The extracted DNA was quantified using a GeneQuant Pro spectrophotometer (Amersham, Buckinghamshire, UK), and then diluted to $20 \mathrm{mg} \cdot \mathrm{L}^{-1}$ with $0.1 \mathrm{X}$ TE buffer.

\section{Phenotypic assessment and fruit harvest}

Foliage color (red or green) was assessed visually for all progeny in the 'Fuji' $\times$ 'Maypole' and 'Tsugaru' $\times$ 'Maypole' populations. Fruit samples were harvested over 6 successive years from 2006 to 2011. Fruit from individual genotypes were picked at maturity when the ground color at the calyx end had changed from green to yellowish green or creamy and immediately before commencement of fruit drop in mid-August to late October. The fruits in each genotype were harvested several times at maturity and the harvest date is the average date. Days to fruit maturity are shown as the number of days from full bloom date to harvest date. Fruit skin color (red or yellow skin, and striped or nonstriped coloration) and fruit flesh color (red or white) were also assessed visually for fruiting progeny in 'Fuji' $\times$ 'Maypole' and 'Tsugaru' $\times$ 'Maypole' populations. The skin coloring pattern was evaluated after pigmentation in fruit skin to avoid misclassification, where clear differences between striped and non-striped skin traits were observed.

\section{MdMYB10 promoter and MdMYBA analysis}

$M d M Y B 10$ promoter region genotypes ( $\mathrm{R} 1$ or $\mathrm{R} 6$ ) were analyzed using MdMYB10 marker (Espley et al., 2009). Polymerase chain reactions (PCR) were conducted in $20 \mu \mathrm{L}$ volumes containing $10 \mathrm{mM}$ Tris- $\mathrm{HCl}$ (pH 8.3), $50 \mathrm{mM} \mathrm{KCl}, 2.5 \mathrm{mM} \mathrm{MgCl}_{2}, 0.2 \mathrm{mM}$ dNTPs, $5 \mathrm{pM}$ each of forward and reverse primers, $20 \mathrm{ng}$ genomic 
DNA, and 0.5 units of Taq polymerase (Takara, Shiga, Japan). The reaction mixture was then overlaid with two drops of mineral oil. PCR amplification was performed on an OmniGene HB-TR3-CM programmable thermal cycler (Hybaid, Middlesex, UK) with conditions as follows: initial denaturation at $94^{\circ} \mathrm{C}$ for $2 \mathrm{~min}$ and $45 \mathrm{~s}$, followed by 40 cycles at $94^{\circ} \mathrm{C}$ for $55 \mathrm{~s}, 55^{\circ} \mathrm{C}$ for $55 \mathrm{~s}$, and $72^{\circ} \mathrm{C}$ for $1 \mathrm{~min} 39 \mathrm{~s}$, and final extension at $72^{\circ} \mathrm{C}$ for $10 \mathrm{~min}$. The amplified products were separated on a $6 \%$ denaturing polyacrylamide gel containing $8 \mathrm{M}$ urea in $0.5 \mathrm{X}$ TBE buffer. Fragments were visualized by silverstaining using a Silver Sequence DNA Sequencing System (Promega, Madison, WI, USA).

MdMYBA genotypes were analyzed using MdMYBA marker (Ban et al., 2007). PCR amplification was carried out under the following conditions: initial denaturation at $94^{\circ} \mathrm{C}$ for $4 \mathrm{~min}$, followed by 30 cycles at $94^{\circ} \mathrm{C}$ for $1 \mathrm{~min}, 53^{\circ} \mathrm{C}$ for $1 \mathrm{~min}$, and $72^{\circ} \mathrm{C}$ for $1 \mathrm{~min}$, and final extension at $72^{\circ} \mathrm{C}$ for $5 \mathrm{~min}$. The amplified products were separated on a $2.0 \%$ agarose gel in $0.5 \mathrm{X}$ TBE buffer. The gels were stained with ethidium bromide $\left(10 \mathrm{~g} \cdot \mathrm{L}^{-1}\right)$ and fragments were detected under UV light.

\section{SSR analysis}

SSR markers located in each linkage group were selected from previously reported markers (Gianfranceschi et al., 1998; Guilford et al., 1997; Hokanson et al., 1998; Liebhard et al., 2002; Silfverberg-Dilworth et al., 2006; Yamamoto et al., 2002), and each primer was labeled with fluorescent dye (6-FAM, HEX, or NED). PCR amplifications were carried out as follows: initial denaturation at $94^{\circ} \mathrm{C}$ for $2 \mathrm{~min}$, followed by 35 cycles at $94^{\circ} \mathrm{C}$ for $1 \mathrm{~min}, 60-55^{\circ} \mathrm{C}$ for $1 \mathrm{~min}$, and $72^{\circ} \mathrm{C}$ for $2 \mathrm{~min}$, and final extension at $72^{\circ} \mathrm{C}$ for $8 \mathrm{~min}$. The allele sizes of parental amplicons were determined using an ABI PRISM 310 Automated Fluorescent DNA Sequencer (Applied Biosystems, Foster City, Canada) and the amplified products from the population were separated on a $6 \%$ denaturing polyacrylamide gel and visualized by silver staining.

\section{Md-Rf marker analysis}

Md-Rf genotypes, known to be linked to the gene for fruit skin color, were analyzed using Md-Rf markers (Cheng et al., 1996). PCR amplification was carried out as follows: initial denaturation at $94^{\circ} \mathrm{C}$ for $3 \mathrm{~min}$, followed by 40 cycles at $94^{\circ} \mathrm{C}$ for $30 \mathrm{~s}, 55^{\circ} \mathrm{C}$ for $1 \mathrm{~min}$, and $72^{\circ} \mathrm{C}$ for $1 \mathrm{~min}$. The amplified products were separated on a $2.0 \%$ agarose gel.

\section{Construction of genetic linkage maps and comparison with the apple genome}

Segregation analysis based on the pseudo-test cross mapping strategy (Grattapaglia and Sederoff, 1994) was performed on 79 progeny of 'Fuji' $\times$ 'Maypole'. The segregation of each marker was scored by the presence or absence of specific parental bands, and those markers segregating in a $1: 1$ ratio $(>0.05)$ were used for linkage analysis. Genetic linkage maps including SSRs, STS markers, and some phenotypes were constructed using MAPL98 mapping software (Ukai, 1998). Phenotypes were abbreviated as follows: red leaf/red flesh $(R l / R f l)$ and red striped skin $(R s t)$. Genetic map distances in centi-Morgans (cM) were calculated using the Kosambi mapping function (Kosambi, 1944).

The locations of the markers mapped by linkage analysis were confirmed on the cv. 'Golden Delicious' genomic sequence using the Genome Database for Rosaceae (GDR) NCBI BLAST server (http:// www.rosaceae.org/). The primer sequences of markers located on linkage maps were used as the search queries.

\section{QTL analysis and candidate gene searching}

QTL analyses were carried out using all markers mapped onto genetic linkage maps of 'Fuji' and 'Maypole'. Windows QTL cartographer version 2.5 (Wang et al., 2010) was used to perform the composite interval mapping (CIM) method with a step size of $1.0 \mathrm{cM}$. The LOD threshold for declaring the presence of a QTL was defined by the 1000 permutation test at $5 \%$ level of significance. The position at which the LOD score curve reached its maximum was used as the estimate of the QTL location. The percentage of the phenotypic variance explained by a QTL was estimated as the coefficient of determination $\left(R^{2}\right)$.

A list of predicted gene transcripts within the QTL interval was extracted from the apple cv. 'Golden Delicious' genomic sequence using GDR GBrowse. Gene locations and putative functions were based on homology to known Arabidopsis proteins obtained from The Arabidopsis Information Resource (TAIR, http:// www.arabidopsis.org/).

\section{Results}

\section{Phenotypic segregation of several coloration traits}

Each leaf color, flesh color, skin color, and skin coloring pattern phenotype observed in the 'Fuji' $\times$ 'Maypole' population segregated in the expected $1: 1$ or $3: 1$ ratio, as confirmed by the $\chi^{2}$ test (Table 1 ). With regard to skin coloration, three different phenotypes were observed in the progeny: red skin/striped, red skin/nonstriped, and yellow skin (Fig. 1). Among these phenotypes, skin color traits segregated in a $3: 1$ ratio (red : yellow), implying that both 'Fuji' and 'Maypole' are heterozygous for red fruit skin $(R f s)$. However, skin coloring patterns segregated in a 1:1 ratio (striped:nonstriped), suggesting that 'Fuji' is heterozygous for dominant striped skin (Rst), whereas 'Maypole' is homozygous for the non-striped skin trait. Red leaf $(R l)$ and red flesh $(R f l)$ traits derived from 'Maypole' cosegregated $\left(\chi^{2}=0.06\right)$ without recombination. 
Table 1. Phenotype segregation of foliage color and fruit coloring traits in the progeny of 'Fuji' $\times$ 'Maypole'.

\begin{tabular}{|c|c|c|c|c|c|c|c|}
\hline \multirow{2}{*}{ Phenotype } & \multicolumn{2}{|c|}{ Parental genotype ${ }^{z}$} & \multirow{2}{*}{$\begin{array}{l}\text { Total number } \\
\text { of progeny }\end{array}$} & \multicolumn{2}{|c|}{ Phenotype Segregation } & \multirow{2}{*}{$\chi^{2}$} & \multirow{2}{*}{$P$} \\
\hline & Fuji & Maypole & & Expected & Observed & & \\
\hline Foliage color (red, green) & $r l \cdot r l$ & $R l \cdot r l$ & 79 & $1: 1(R l: r l)$ & $42: 37(R l: r l)$ & 0.32 & 0.57 \\
\hline Flesh color (red, white) & $r f l \cdot r f l$ & $R f l \cdot r f l$ & 72 & $1: 1(R f l: r f l)$ & $37: 35(R f l: r f l)$ & 0.06 & 0.81 \\
\hline Skin color (red, yellow) & $R f s^{s} \cdot r f s$ & $R f s^{n s} \cdot r f s$ & 72 & $3: 1\left(R f s^{s+n s}: r f s\right)$ & $52: 20\left(R f s^{s+n s}: r f s\right)$ & 0.30 & 0.59 \\
\hline Skin coloring type (stripe, non-stripe) & Rst $\cdot r s t$ & $r s t \cdot r s t$ & 72 & $1: 1(R s t: r s t)$ & $31: 41$ (Rst:rst) & 1.38 & 0.24 \\
\hline
\end{tabular}

${ }^{\mathrm{z}} R l$ : Red leaf, $R f l$ : Red flesh, $R f s^{s}$ : Red fruit skin (striped), $R f s^{n s}$ : Red fruit skin (non-striped), Rst: Red striped skin

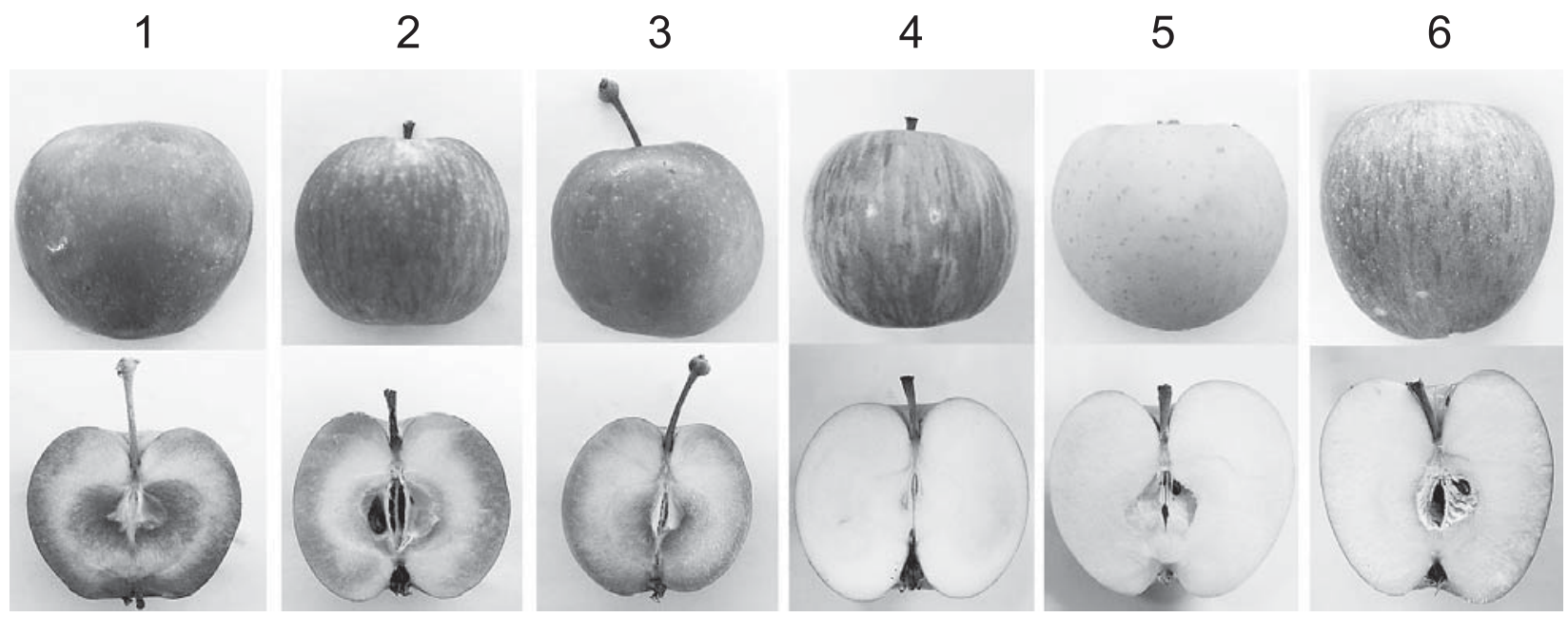

Fig. 1. Phenotypic segregation of fruit skin and fruit flesh color in the progeny of 'Fuji' $\times$ 'Maypole'. 1: 'Maypole'; 2: Red striped skin and red flesh; 3: Red non-striped skin/red flesh; 4: Red striped skin and white flesh; 5: Yellow skin/white flesh; 6: 'Fuji'.

\section{Identification of MdMYB10 promoter and MdMYBA genotypes}

Amplification of the MdMYB10 promoter region of 'Fuji' yielded a single 392-bp band, whereas two bands of $496 \mathrm{bp}$ and $394 \mathrm{bp}$, the latter a little larger than the band from 'Fuji', were generated from 'Maypole'. These fragments showed polymorphism in the progeny of 'Fuji' $\times$ 'Maypole'. With regard to alleles derived from 'Maypole', the 496-bp fragment, corresponding to the R6 promoter derived from 'Baskatong' (Nocker et al., 2011), was observed only in the red leaf/red flesh progeny, whereas the 394-bp fragment, derived from 'Wijcik McIntosh', was detected in the green leaf/white flesh offspring (Fig. 2a). With respect to alleles from 'Fuji', the 392-bp fragment and a missing fragment (null allele) were observed in the progeny. This polymorphism was present in a $1: 1$ ratio $\left(\chi^{2}=1.53\right)$. The 392-bp fragment was detected only in the striped skin progeny, whereas no fragment was found in progeny with nonstriped or yellow skin traits. These results suggest that one of the MdMYB10 promoter regions of 'Fuji' is likely to be the null allele, and that MdMYB10 promoter genotypes are related to skin coloring patterns as well as fruit flesh color.

In MdMYBA analysis, a 723-bp fragment and a 656-bp fragment, which correspond to red skin and noncoloring traits, respectively, were identified in 'Fuji', but only a
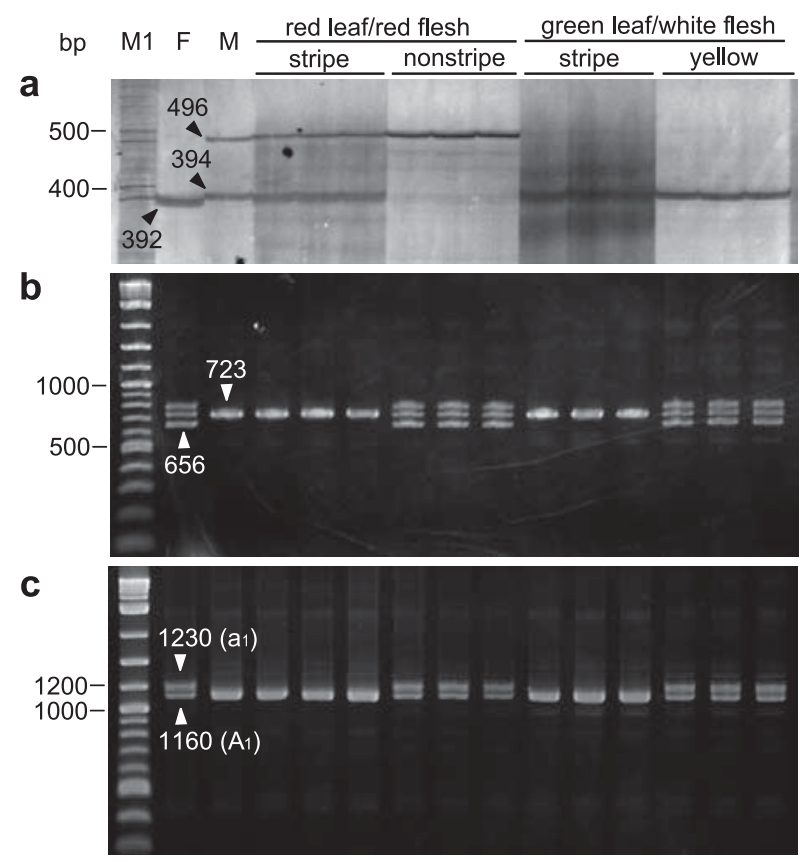

Fig. 2. Amplification of MdMYB10 promoter, MdMYBA and MdRf markers in the progeny of 'Fuji' × 'Maypole'. a: MdMYB10 promoter. b: MdMYBA marker. c: Md-Rf marker. F: 'Fuji', M: 'Maypole', M1: ladder marker. 
723-bp fragment was detected in 'Maypole' (Fig. 2b). Banding patterns generated from 'Fuji' $\times$ 'Maypole' progeny were either a single 723-bp fragment (homozygote) or a pair of 723-bp and 656-bp fragments (heterozygote) that were present in a ratio corresponding to a $1: 1$ segregation ratio $\left(\chi^{2}=1.53\right)$. The 723 -bp and 656-bp fragments derived from 'Fuji' (Rst/rst) cosegregated with striped skin and non-striped or yellow skin traits, respectively, so that the $M d M Y B A$ genotypes of 'Fuji' coincided with the 392-bp fragment (and null allele) amplified in the MdMYB10 promoter region (Fig. 2a, b); however, the 723-bp fragment derived from Maypole $(r s t / r s t)$ was not associated with the striped skin trait. MdMYBA genotypes of 'Maypole' showed no polymorphism with respect to MdMYB10 promoter genotypes, because all progeny had only the 723-bp fragment.

\section{Md-Rf analysis}

A 1160-bp fragment $\left(\mathrm{A}^{1}\right)$, known to be linked to the red skin trait, and a 1230-bp fragment $\left(\mathrm{a}^{1}\right)$, linked to the noncoloring trait, were amplified in 'Fuji'; only a 1160-bp fragment $\left(\mathrm{A}^{1}\right)$ was generated from 'Maypole' (Fig. 2c). In the $\mathrm{F}_{1}$ population, homozygote $\left(\mathrm{A}^{1} \mathrm{~A}^{1}\right)$ and heterozygote $\left(\mathrm{A}^{1} \mathrm{a}^{1}\right)$ progeny with the 1160 -bp fragment segregated in a $1: 1$ ratio $\left(\chi^{2}=0.38\right)$. These results demonstrated that 'Fuji' was heterozygous for the 1160-bp allele $\left(\mathrm{A}^{1}\right)$, whereas 'Maypole' was homozygous for the $\mathrm{A}^{1}$ allele. The $\mathrm{A}^{1}$ and $\mathrm{a}^{1}$ alleles of 'Fuji' $(R s t / r s t)$ corresponded to striped skin and non-striped or yellow skin traits, respectively, whereas the $A_{1}$ alleles of Maypole $(r s t / r s t)$ were not associated with the striped skin trait (Fig. 2c). Because recombination between Md$\mathrm{Rf}$ and the striped skin trait (Rst) was found in only two of 79 individual offspring, the genetic distance was estimated to be $2.6 \mathrm{cM}$, indicating that $\mathrm{Md}-\mathrm{Rf}$ is tightly linked to $M d M Y B A$.

\section{Genetic analysis of fruit maturity in progeny of 'Maypole'}

Number of days to fruit maturity was assessed with 'Fuji' $\times$ 'Maypole' and 'Tsugaru' $\times$ 'Maypole' populations. For each genotype, average phenotypic value of 6 years from 2006 to 2011 was used in genetic analysis, because fruiting in two population was not stable among years and a limited number of genotypes produced fruits in each year (e.g., in 2006, fruits of 34 genotypes were harvested from 'Fuji' $\times$ 'Maypole' population). Although the harvest date of the fruits in each genotype varied among years depending on the flowering time, the number of days to fruit maturity was almost constant.

In the 'Fuji' $\times$ 'Maypole' population, the frequency distribution of days to fruit maturity in each progeny showed continuous distribution ranging from 110 to 180 days after full bloom (DAFB). When the frequency distribution in the 'Fuji' $\times$ 'Maypole' population was compared with each coloration trait, fruits of red leaf/ red flesh progeny tended to have earlier harvest dates, ranging from 110 to $155 \mathrm{DAFB}$, than those of green leaf/white flesh pogeny, which ranged from 126 to 180 DAFB (Fig. 3). This tendency for fruit maturity was also found in every year investigated (data not shown). In contrast to the 'Fuji' $\times$ 'Maypole' population, no clear difference was observed between red and green progeny in the 'Tsugaru' $\times$ 'Maypole' population, which exhibited a distribution ranging from 117 to 145 DAFB. These results indicate that 'Maypole' and 'Tsugaru' possess a major factor responsible for early fruit maturation, with the factor derived from 'Maypole' appearing to be tightly linked to the red leaf/red flesh trait.

\section{Mapping of coloration traits and candidate genes}

A total of 120 genome-wide SSR markers were used to construct genetic linkage maps for 'Fuji' and 'Maypole'. The 'Fuji' map consisted of 88 markers ordered in 17 linkage groups. The map defined $932.3 \mathrm{cM}$ of total map distance and had an average distance between adjacent markers of $7.8 \mathrm{cM}$. The 'Maypole' map is based on 77 markers on 17 linkage groups. The map defined $894.9 \mathrm{cM}$ of total map distance and had an
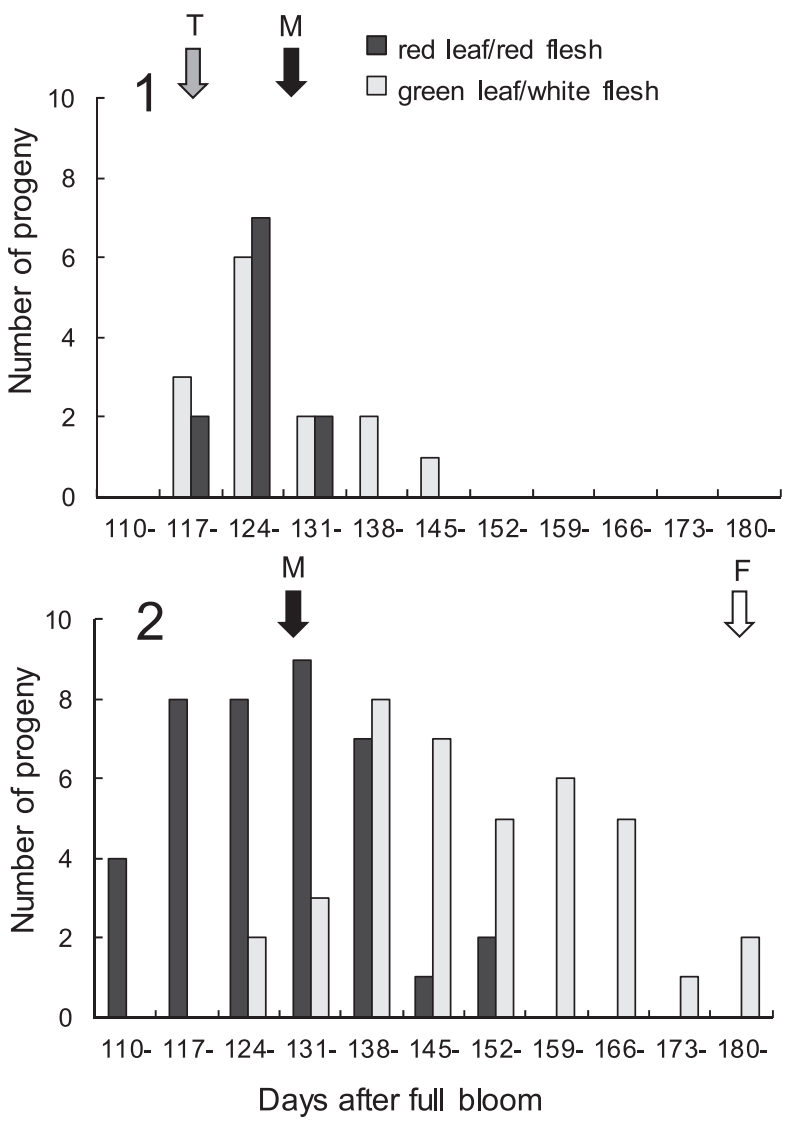

Fig. 3. Frequency distributions of number of days to fruit maturity in $F_{1}$ populations of (1) 'Tsugaru' $\times$ 'Maypole' and (2) 'Fuji' $\times$ 'Maypole'. Phenotypic value of each genotype represents the mean of 6 successive years from 2006 to 2011. The black, white, and gray arrows indicate harvest dates of 'Maypole' (M), 'Fuji' (F), and 'Tsugaru' (T), respectively. 
average distance between adjacent markers of $8.2 \mathrm{cM}$.

LG 9 of 'Fuji' consisted of 10 markers with a total length of $43.9 \mathrm{cM}$, whereas that of 'Maypole' comprised 8 markers spanning $58.3 \mathrm{cM}$ (Fig. 4). The red leaf/red flesh trait $(R l / R f l)$ and $M d M Y B 10$ mapped onto the bottom of LG 9 in 'Maypole', and co-segregated with SSR marker CN444542. The red striped skin trait $(R s t)$ and $M d M Y B A$ were located in the same region of LG 9 in 'Fuji', and also co-segregated with CN444542. The constructed maps were usable for comparison with the previously published Malus ('Fiesta' $\times$ 'Discovery') reference map (Silfverberg-Dilworth et al., 2006, data not shown) and with physical maps derived from the cv. 'Golden Delicious' genomic sequence (Velasco et al., 2010).

At least two R2R3-type $M Y B$ genes were found in the region of the CN444542 marker (29.43 Mbp) on the apple genome. The first $M Y B$ gene (MYB gene 1: MDP0000259614) showed $100 \%$ and $98 \%$ similarity at the nucleotide sequence level with $M d M Y B A$ (Ban et al., 2007) and MdMYB10 (Espley et al., 2007), respectively, whereas the second gene (MYB gene 2: MDP0000573302) exhibited lower similarity $(<80 \%)$ at both nucleotide and amino acid sequence levels.

\section{QTL analysis for fruit maturity}

QTL analysis was performed using the average phenotypic value for the 6 years investigated. A single major QTL $(\mathrm{LOD}=8.94)$ for the earliness of fruit maturity was detected near the MdMYB10 locus in 'Maypole' (Fig. 5). This QTL explained 35.6\% of the

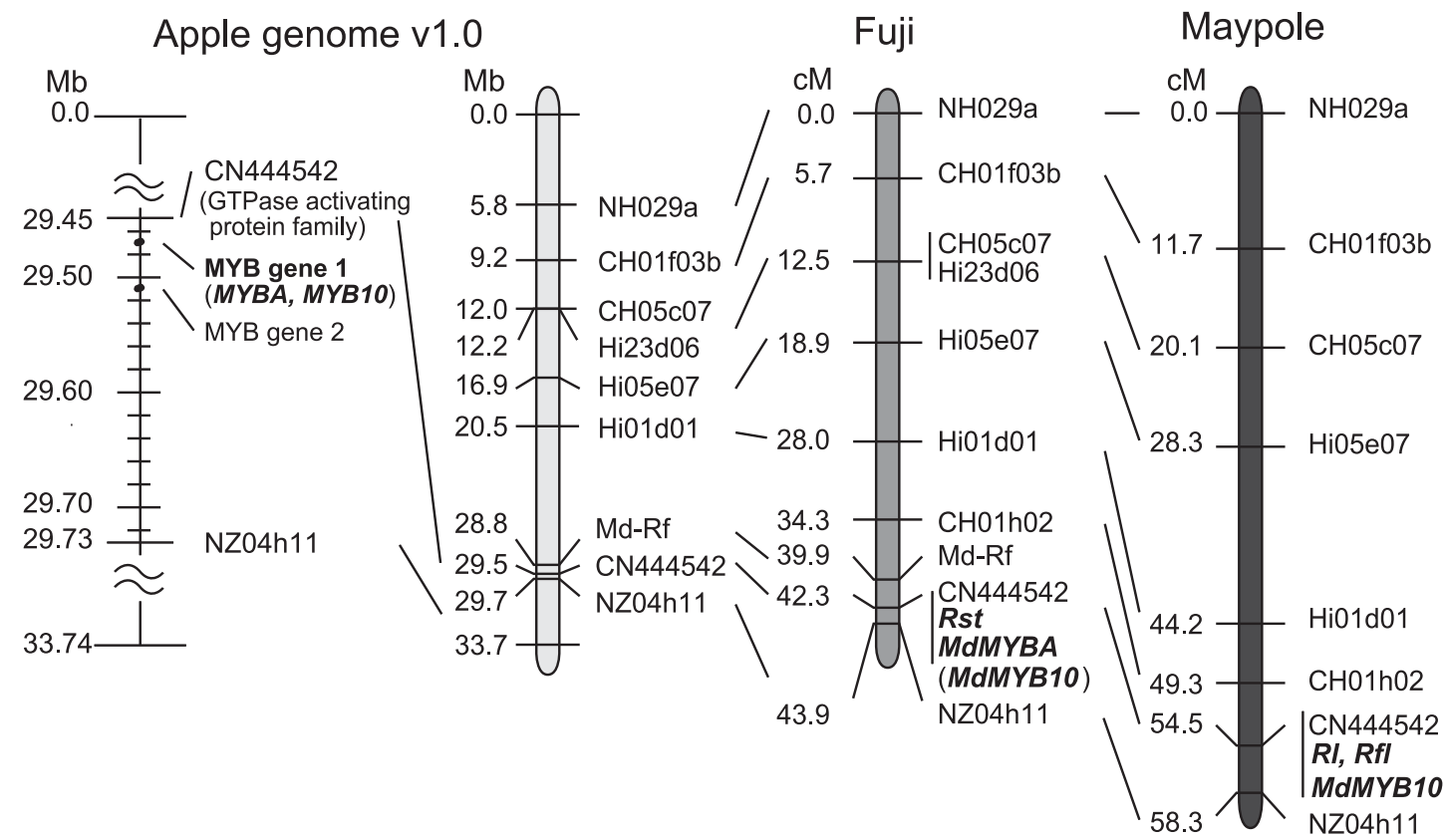

Fig. 4. Linkage group 9 of 'Fuji' and 'Maypole' and physical map of chromosome 9 from the apple genome v1.0. Genetic map distance in 'Fuji' and 'Maypole' represented in centiMorgans (cM). Rst: Red striped skin; Rl: Red leaf; Rfl: Red flesh.

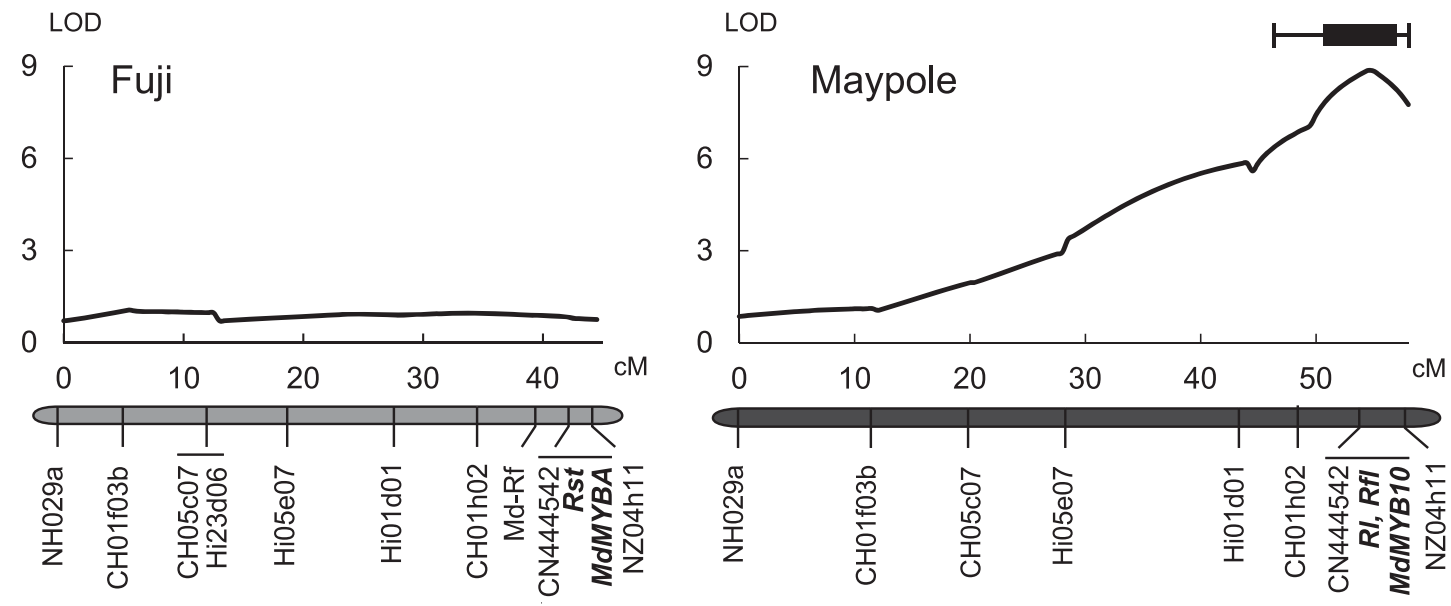

Fig. 5. LOD profile of the QTL for fruit maturity (2006-2011) in linkage group 9 of 'Fuji' and 'Maypole'. Solid black and thin bars indicate 1LOD and 2-LOD support intervals for the QTL position (maximum LOD score), respectively. 
phenotypic variance in the 'Fuji' $\times$ 'Maypole' population. From this result, we attempted to perform QTL analysis using the phenotypic value for each year from 2006 to 2011. The significant QTL tested by the 1000 permutation test at $5 \%$ level of significance was also detected near the MdMYB10 locus in each year, although the phenotypic variance fluctuated from $15.0 \%$ (2010) to $59.9 \%$ (2006). In contrast to the LG 9 of 'Maypole', no significant QTLs were detected in LG 9 of 'Fuji' and in the other linkage groups of both cultivars.

\section{Candidate gene searching for fruit maturity}

To elucidate the genetic mechanisms underlying the earliness of fruit maturity associated with MdMYB10 and/or the red flesh trait, the region around the MdMYB10 locus $(29.47 \mathrm{Mbp})$ on the apple genome was examined for candidate genes possibly related to fruit maturity using in silico analysis. Within the $28.00-31.00 \mathrm{Mbp}$ region corresponding to the QTL interval defined in LG 9 of 'Maypole', there were 427 predicted genes. Based on the apple genome annotation, an ACC synthase, known to be a key enzyme controlling ethylene biosynthesis, was found $30.50 \mathrm{Mbp}$ downstream from MdMYB10 (MdMYBA). This ACC synthase, however, exhibited low sequence homology with the already identified ACSs in apple (accession numbers, MdACS1: L31347; MdACS2: U73815; MdACS3: U73816; MdACS5A: AB034992; MdACS5B: AB034993). Several candidate genes, including pectin esterase and late embryogenesis abundant (LEA) genes, gibberellinassociated genes (gibberellin-regulated protein and RGA-Like), and auxin-associated genes were also located in this region.

\section{Discussion}

Genetic analysis for coloration traits and candidate genes

Red leaf and red flesh traits in apple have previously been shown to co-segregate with MdMYB10, and red leaf/red flesh varieties contain five direct tandem repeats of a 23-bp sequence in the MdMYB10 promoter region (R6 promoter) (Espley et al., 2007, 2009). In the 'Fuji' × 'Maypole' population, all red-fleshed fruits were harvested from red-leaved progeny; these red leaf/red flesh progeny contained the R6 promoter (496 bp), confirming that the red leaf/red flesh trait in 'Maypole' is controlled by MdMYB10. Of the 'Fuji' alleles, a 392-bp fragment ( $\mathrm{R} 1$ promoter) was shown to correspond to the red striped skin trait $(R s t)$, and the other null allele to yellow skin or non-striped skin. Espley et al. (2009) reported that 'Fuji' contains the R1 promoter (392 bp), but not its genotype, suggesting that a deletion or a mutation at the primer annealing site in the MdMYB10 promoter region may have occurred in 'Delicious' or 'Ralls Janet'.

Recent studies indicated that MdMYB10 and $M d M Y B A$, the latter of which is responsible for skin coloration, are likely to be allelic in the apple genome (Lin-Wang et al., 2010, 2011). In the present study, we analyzed the relationship between MdMYB10 and $M d M Y B A$ along with their association with coloration phenotypes on the same genetic material. MdMYBA genotyping detected a 723-bp fragment and a 656-bp fragment in 'Fuji', whereas 'Maypole' was homozygous for the 723-bp fragment. Among these fragments, the 723-bp fragment from 'Fuji' co-segregated with red striped skin, while the 656-bp fragment co-segregated with non-striped skin or yellow skin. These genotypes therefore correspond to the MdMYB10 promoter genotypes of R1 (392 bp) and the null allele of 'Fuji'. Wilcox and Angelo (1936) showed that by classifying seedlings into striped and non-striped, which included blushed and unpigmented fruits, the striping trait behaved as a single dominant gene, indicating that $M d M Y B A$ is the candidate gene for the skin coloring pattern. Furthermore, Telias et al. (2011) reported that apple skin patterning might be associated with the differential expression of MdMYB10, resulting from variations in the degree of methylation in the MdMYB10 promoter region. Methylation and/or the null allele in the MdMYB10 promoter region of 'Fuji' may be associated with $M d M Y B 10$ (MdMYBA) expression.

$M d M Y B A$ loci in 'Maypole' were homozygous for a 723-bp fragment with no polymorphism; however, in MdMYB10 promoter genotypes, 'Maypole' has a 496-bp (R6) allele corresponding to a red skin phenotype derived from 'Baskatong' and a 394-bp allele associated with a yellow skin phenotype from 'Wijcik McIntosh'. In a previous study (Espley et al., 2009), MdMYB10 markers amplified a 392-bp fragment from the R1 promoter, the same size as that detected in the MdMYB10 promoter of 'Fuji' but smaller than that of 'Maypole' (394 bp). These results imply that a mutation in the promoter region carrying the 394-bp fragment may have occurred in the MdMYB10 allele of 'Maypole', resulting in loss of expression or function in the MdMYBA allele. Meanwhile, Ban et al. (2007) have reported that the 723-bp fragment of MdMYBA marker corresponds to both $\mathrm{A}^{1}$ and $\mathrm{a}^{2}$ allele amplified by Md-Rf marker (where $\mathrm{A}^{1}$ and $\mathrm{a}^{2}$ are linked to red and yellow skin color, respectively). In this case, discrimination of the $\mathrm{A}^{1}$ and $\mathrm{a}^{2}$ genotype is impossible by $M d M Y B A$ marker. $M d M Y B A$ loci in 'Maypole' therefore may be heterozygous for $\mathrm{A}^{1}$ and $\mathrm{a}^{2}$ alleles.

The red leaf/red flesh trait of 'Maypole' and its candidate gene, MdMYB10, and the red striped skin trait and $M d M Y B A$ of 'Fuji' were located in the same region at the bottom of LG 9 of each cultivar; these traits or genes co-segregated with the SSR marker CN444542. Moreover, the CN444542 primer sequence exhibited $100 \%$ similarity to the bottom of chromosome 9 $(29.43 \mathrm{Mb})$ in the apple genome (Velasco et al., 2010), and a candidate gene identical in sequence to $M d M Y B A$ (Ban et al., 2007) was found in this region $(29.47 \mathrm{Mb})$. 
None of the other candidate genes for MdMYB10 or $M d M Y B A$, however, have been located at this locus in the apple genome assembly, indicating that MdMYB10 and MdMYBA are probably allelic. This conclusion is further supported by the fact that the MdMYB10 promoter genotype detected in 'Fuji' and 'Maypole' co-segregated not only with fruit flesh color but also with the MdMYBA genotype or skin coloring pattern.

Cheng et al. (1996) have reported that Md-Rf genotypes of 'Baskatong' and 'McIntosh' are $\mathrm{A}^{1} \mathrm{~A}^{1}$ and $A^{1} n$, respectively (where $n$ is the null allele linked to yellow skin). Interestingly, although all progeny of 'Fuji' $\times$ 'Maypole' carried the $\mathrm{A}^{1}$ allele known to be linked to the red skin trait, some of them produced yellow-skinned fruit. If recombination between Md-Rf and the skin color trait had not occurred in 'Wijcik McIntosh', the null allele linked to the yellow skin phenotype would have been detected in any 'Fuji' $\times$ 'Maypole' progeny with yellow skin. The Md-Rf null allele was not inherited, however, by 'Maypole', which was $\mathrm{A}^{1} \mathrm{~A}^{1}$. There must therefore have been recombination between Md-Rf and the skin color trait in the maternal parent 'Wijcik McIntosh', because evidence for such a recombination was found in this study.

\section{QTL analysis for fruit maturity}

Genetic analysis of the relationship between the red flesh trait and fruit maturity in the 'Fuji' $\times$ 'Maypole' population uncovered clear differences, with fruits of red-fleshed progeny tending to mature earlier than those of white-fleshed progeny. This suggests that a major factor responsible for fruit maturation is linked to the red leaf/red flesh trait in 'Maypole'. In addition, a major QTL explaining 35.6\% of the phenotypic variance (LOD $=8.94)$ was detected at the MdMYB10 locus in 'Maypole'. A significant QTL was also detected near the MdMYB10 locus in each year from 2006 to 2011, indicating that this QTL seems to be stable over 6 successive years. This result provides preliminary data, however, because a limited number of genotypes produced fruits in each year $(n=34-61)$. The reason why phenotypic variance explained by the QTL fluctuated among years is probably due to the number of genotypes fruited. Indeed, in 2006, when the maximal LOD score (15.27) and $R^{2}$ (59.9) were obtained, fruited progeny were only 34 genotypes in the 'Fuji' $\times$ 'Maypole' population, and in this case, distorted frequency distribution may have caused an overestimate of total phenotypic variance in QTL analysis.

Previous QTL studies in apple have identified several minor QTLs for fruit maturity, accounting for 9 to $17 \%$ of the total variance (Kenis et al., 2008; Liebhard et al., 2002). Kenis et al. (2008) identified a QTL with a small effect associated with $10.4 \%$ of the phenotypic variance $(\mathrm{LOD}=4.50)$ in the same region at the bottom of LG 9 of 'Maypole', but this QTL was detected during only one of two seasons investigated. The major QTL detected in the present study, therefore, may be specific for the red leaf/red flesh trait in 'Maypole'.

To search for the possible factors responsible for fruit maturity, we analyzed MdACS1, known to regulate ethylene biosynthesis (Harada et al., 2000; Sato et al., 2004), in 'Fuji' × 'Maypole' progeny (data not shown). The genotype in all the offspring was heterozygous because 'Fuji' and 'Maypole' are homozygous for ACS1 2 and ACS1-1, respectively. However, on the apple genome, an unknown ACS was found within a QTL interval identified in LG 9, suggesting the possibility of a specific ethylene biosynthetic pathway for the red leaf/ red flesh trait. Gibberellin- and auxin-related genes supposedly related to fruit growth, as well as LEA and pectin esterase genes associated with seed and fruit maturity, respectively, were also located in this interval, although no quantitative trait gene (QTG) was identified.

Other reported research, however, allows us to propose a hypothesis regarding the relationship between the red flesh trait and fruit maturity. A relatively recent genomewide duplication resulted in the transition from nine ancestral chromosomes to 17 chromosomes in the Pyreae, which includes Malus species (Velasco et al., 2010). Chromosome 9, which carries the MdMYB10 locus, is homologous with chromosome 17 , and a high level of synteny, including $M d M Y B$ genes, is observed between those chromosomes. QTLs for the earliness of fruit maturity could not be detected in LG 17 of 'Fuji', 'Maypole', and other reports (Kenis et al., 2008; Liebhard et al., 2002), so the QTG may be specific to chromosome 9. From this hypothesis, the expression of MdMYB10 and/or increased ectopic synthesis or accumulation of anthocyanins in fruit flesh might lead to factors for early fruit maturation. Indeed, many previously-released red-fleshed varieties, including 'Geneva', 'Redfield', and 'Almata', are known to be early-maturing cultivars. Furthermore, our recent data showed a significant correlation $\left(r=-0.66^{* *}\right.$, unpublished data) between the harvest date and fruit flesh red coloration intensity in 'Fuji' $\times$ 'Maypole' progeny; this suggests that elevated anthocyanin levels in red flesh fruit might promote fruit maturation. Moreover, the QTL for fruit maturity may be detected in LG 17 of 'Pink Pearl' because the red flesh trait in this variety is due to a dominant allele linked to the $S$-locus (Sekido et al., 2010). The hypotheses described above could be evaluated by analyzing the expression patterns of the candidate genes identified in the present study and the relationship between MdMYB10 expression (intensity of red coloration in fruit flesh) and fruit harvest date.

\section{Literature Cited}

Ban, Y., C. Honda, Y. Hatsuyama, M. Igarashi, H. Bessho and T. Moriguchi. 2007. Isolation and functional analysis of a MYB trancription factor gene that is a key regulator for the development of red coloration in apple skin. Plant Cell Physiol. 48: 958-970. 
Chagne, D., C. M. Carlisle, C. Blond, R. K. Volz, C. J. Whitworth, N. C. Oraguzie, R. N. Crowhurst, A. C. Allan, R. V. Espley, R. P. Hellens and S. E. Gardiner. 2007. Mapping a candidate gene (MdMYB10) for red fresh and foliage colour in apple. BMC Genomics 8: 212-222.

Cheng, F. S., N. F. Weeden and S. K. Brown. 1996. Identification of co-dominant RAPD markers tightly linked to fruit skin color in apple. Theor. Appl. Genet. 93: 222-227.

Eberhardt, M. V., C. Y. Lee and R. H. Liu. 2000. Nutrition: Antioxdant activity of fresh apples. Nature 405: 903-904.

Espley, R. V., C. Brendolise, D. Chagne, S. Kutty-Amma, S. Green, R. Volz, J. Putterill, H. J. Schouten, S. E. Gardiner, R. P. Hellens and A. C. Allan. 2009. Multiple repeats of a promoter segment causes transcription factor autoregulation in red apples. Plant Cell 21: 168-183.

Espley, R. V., R. P. Hellens, J. Putterill, D. E. Stevenson, S. KuttyAmma and A. C. Allan. 2007. Red coloration in apple fruit is due to the activity of the MYB transcription factor, MdMYB10. Plant J. 49: 414-427.

Gao, J. J., Z. Zhang, R. H. Peng, A. S. Xiong, J. Xu, B. Zhu and Q. H. Yao. 2011. Forced expression of Mdmyb10, a myb transcription factor gene from apple, enhances tolerance to osmotic stress in transgenic Arabidopsis. Mol. Biol. Rep. 38: 205-211.

Gianfranceschi, L., N. Seglias, R. Tarchini, M. Komjanc and C. Gessler. 1998. Simple sequence repeats for the genetic analysis of apple. Theor. Appl. Genet. 96: 1069-1076.

Grattapaglia, D. and R. Sederoff. 1994. Genetic linkage maps of Eucalyptus grandis and Eucalyptus urophylla using a pseudotestcross: mapping strategy and RAPD markers. Genetics 137: 1121-1137.

Guilford, P., S. Prakash, J. M. Zhu, E. Rikkerink, S. Gardiner, H. Bassett and R. Forster. 1997. Microsatellites in Malus $\times$ domestica (apple): abundance, polymorphism and cultivar identification. Theor. Appl. Genet. 94: 249-254.

Harada, T., T. Sunako, Y. Wakasa, J. Soejima, T. Satoh and M. Niizeki. 2000. An allele of the 1-aminocyclopropane-1carboxylate synthase gene (MdACS1) accounts for the low level of ethylene production in climacteric fruits of some apple cultivars. Theor. Appl. Genet. 101: 742-746.

Hokanson, S. C., A. K. Szewc-McFadden, W. F. Lamboy and J. R. McFerson. 1998. Microsatellite (SSR) markers reveal genetic identities, genetic diversity and relationships in a Malus $\times$ domestica Borkh. core subset collection. Theor. Appl. Genet. 97: 671-683.

Kenis, K. and J. Keulemans. 2007. Study of tree architecture of apple (Malus $\times$ domestica Borkh.) by QTL analysis of growth traits. Mol. Breed. 19: 193-208.

Kenis, K., J. Keulemans and M. W. Davey. 2008. Identification and stability of QTLs for fruit quality traits in apple. Tree Genet. Genomes 4: 647-661.

King, G. J., J. R. Lynn, C. J. Dover, K. M. Evans and G. B. Seymour. 2001. Resolution of quantitative trait loci for mechanical measures accounting for genetic variation in fruit texture of apple (Malus pumila Mill.). Theor. Appl. Genet. 8: $1227-1235$.

Kosambi, D. D. 1944. The estimation of map distances from recombination values. Ann. Eugen. 12: 172-175.

Liebhard, R., L. Gianfranceschi, B. Koller, C. D. Ryder, R. Tarchini, E. Van De Weg and C. Gessler. 2002. Development and characterisation of 140 new microsatellites in apple
(Malus $\times$ domestica Borkh.). Mol. Breed. 10: 217-241.

Liebhard, R., M. Kellerhals, W. Pfammatter, M. Jertmini and C. Gessler. 2003. Mapping quantitative physiological traits in apple (Malus $\times$ domestica Borkh.). Plant Mol. Biol. 52: 511526.

Lin-Wang, K., K. Bolitho, K. Grafton, A. Kortstee, S. Karunairetnam, T. K. McGhie, R. V. Espley, R. P. Hellens and A. C. Allan. 2010. An R2R3 MYB transcription factor associated with regulation of the anthocyanin biosynthetic pathway in Rosaceae. BMC Plant Biol. 10: 50-66.

Lin-Wang, K., D. Micheletti, J. Palmer, R. Volz, L. Lozano, R. Espley, R. P. Hellens, D. Chagne, D. D. Rowan, M. Troggio, I. Iglesias and A. C. Allan. 2011. High temperature reduces apple fruit colour via modulation of the anthocyanin regulatory complex. Plant cell Environ. 34: 1176-1190.

Nocker, S. V., G. Berry, J. Najdowski, R. Michelutti, M. Luffman, P. Forsline, N. Alsmairat, R. Beaudry, M. G. Nair and M. Ordidge. 2011. Genetic diversity of red-fleshed apples (Malus). Euphytica 185: 281-293.

Sato, T., T. Kudo, T. Akada, Y. Wakasa, M. Niizeki and T. Harada. 2004. Allelotype of a ripening-specific 1aminocyclopropane-1-carboxylate synthase gene defines the rate of fruit drop in apple. J. Amer. Soc. Hort. Sci. 129: 32-36.

Sekido, K., Y. Hayashi, K. Yamada, K. Shiratake, S. Matsumoto, T. Maejima and H. Komatsu. 2010. Efficient breeding system for red-flesh apple based on linkage with $S_{3}$-RNase allele in 'Pink Pearl'. HortScience 45: 534-537.

Silfverberg-Dilworth, E., C. L. Matasci, W. E. Van de Weg, M. P. W. Van Kaauwen, M. Walser, L. P. Kodde, V. Soglio, L. Gianfranceschi, C. E. Durel, F. Costa, T. Yamamoto, B. Koller, C. Gessler and A. Patocchi. 2006. Microsatellite markers spanning the apple (Malus $\times$ domestica Borkh.) genome. Tree Genet. Genomes 2: 202-224.

Takos, A. M., F. W. Jaffe, S. R. Jacob, J. Bogs, S. P. Robinson and A. R. Walker. 2006. Light-induced expression of a $M Y B$ gene regulates anthocyanin biosynthesis in red apples. Plant Physiol. 142: 1216-1232.

Telias, A., K. Lin-Wang, D. E. Stevenson, J. M. Cooney, R. P. Hellens, A. C. Allan, E. E. Hoover and J. M. Bradeen. 2011. Apple skin patterning is associated with differential expression of MYB10. BMC Plant Biol. 11: 93-106.

Ubi, B. E., C. Honda, H. Bessho, S. Kondo, M. Wada, S. Kobayashi and T. Moriguchi. 2006. Expression analysis of anthocyanin biosynthetic genes in apple skin: Effect of UV-B and temperature. Plant Sci. 170: 571-578.

Ukai, Y. 1998. MAPL: A package of computer programs for construction of DNA polymorphism linkage maps and analysis of QTL. Breed. Sci. 45: 139-142.

Velasco, R., A. Zharkikh, J. Affourtit, A. Dhingra, A. Cestaro et al. 2010. The genome of the domesticated apple (Malus $\times$ domestica Borkh.). Nature Genet. 42: 833-839.

Wang, S., C. J. Bsaten and Z. B. Zeng. 2010. Windows QTL Cartographer 2.5. Department of Statistics, North Carolina State University, Raleigh, NC. <http://statgen.ncsu.edu/ qtlcart/WQTLCart.htm>.

Wilcox, A. N. and E. Angelo. 1936. Apple breeding studies, 1: fruit color. Proc. Am. Soc. Hort. Sci. 33: 108-113.

Yamamoto, T., T. Kimura, M. Shoda, Y. Ban, T. Hayashi and N. Matsuta. 2002. Development of microsatellite markers in the Japanese pear (Pyrus pyrifolia Nakai). Mol. Ecol. Notes 2: $14-16$. 